\title{
Is combined peritoneal dialysis and hemodialysis redundant? A nationwide study from Taiwan
}

Mu-Chi Chung ${ }^{1,2,3}$, Tung-Min Yu', Ming-Ju Wu', Ya-Wen Chuang ${ }^{1}$, Chih-Hsin Muo ${ }^{4}$, Cheng-Hsu Chen ${ }^{1}$,
Chao-Hsiang Chang ${ }^{5,6}$, Jeng-Jer Shieh ${ }^{3,7,8}$, Peir-Haur Hung ${ }^{9,10}$, Jein-Wen Chen ${ }^{11,12,13}$ and Chi-Jung Chung ${ }^{14,5^{*}}$ (D)

\begin{abstract}
Background: Combined peritoneal dialysis (PD) and hemodialysis (HD) therapy (combined therapy) has numerous clinical benefits and should be emphasized for PD patients encountering technique failure.

Methods: This 12-year nationwide retrospective study was conducted to compare long-term outcomes (including admission and mortality risks) between combined therapy patients (combined group) and patients directly transferred from PD to HD (transfer group).

Results: All 12,407 incidental PD patients from 2000 to 2010 were enrolled and followed up until the end of 2011. A total of 688 patients in the combined group and 688 patients in the transfer group were selected after 1:1 frequency matching based on age, sex, and PD duration. The overall admission and mortality risks of the two groups were comparable in a Cox proportional hazards model (adjusted hazard ratio $[\mathrm{HR}]=1.06[95 \%$ confidence interval $(\mathrm{Cl})=$ 0.95-1.19] and 1.02 [95\% Cl=0.80-1.30]), respectively). Compared with the transfer group, combined group patients with recent peritonitis or frequent hemodialysis (four HD sessions per month) had significantly higher risk of admission while combined group patients without peritonitis had significantly lower risk. The number of incidents in the combined group increased over time. On average, patients stayed on combined therapy for 2 years.

Conclusions: Combined therapy (two HD sessions per month) is not redundant but a rational and cost-effective treatment, particularly for patients without recent peritonitis. Dialysis staff should be familiar with the advantages and disadvantages of combined therapy and consider it an essential part of integrated dialysis care.
\end{abstract}

Keywords: Peritoneal dialysis, Hemodialysis, Combined therapy, Mortality, Admission, Cohort study

\footnotetext{
* Correspondence: cjchung1010@gmail.com

${ }^{14}$ Department of Public Health, College of Public Health, China Medical

University, No. 91 Hsueh-Shih Road, Taichung 40402, Taiwan

${ }^{15}$ Department of Medical Research, China Medical University Hospital,

Taichung, Taiwan

Full list of author information is available at the end of the article
}

(c) The Author(s). 2020 Open Access This article is licensed under a Creative Commons Attribution 4.0 International License, which permits use, sharing, adaptation, distribution and reproduction in any medium or format, as long as you give appropriate credit to the original author(s) and the source, provide a link to the Creative Commons licence, and indicate if changes were made. The images or other third party material in this article are included in the article's Creative Commons licence, unless indicated otherwise in a credit line to the material. If material is not included in the article's Creative Commons licence and your intended use is not permitted by statutory regulation or exceeds the permitted use, you will need to obtain permission directly from the copyright holder. To view a copy of this licence, visit http://creativecommons.org/licenses/by/4.0/. The Creative Commons Public Domain Dedication waiver (http://creativecommons.org/publicdomain/zero/1.0/) applies to the data made available in this article, unless otherwise stated in a credit line to the data. 


\section{Background}

Peritoneal dialysis (PD) and hemodialysis (HD) are complementary rather than competitive dialysis therapies. PD has benefits as an initial modality due to its association with improved survival during the first 2-3 years [1], lower cost, and greater self-reported quality of life [2]. Accordingly, a policy of integrated dialysis care with PD first and then HD has been established [3]. Technique failure requires $10 \%$ of patients with PD per year to switch to HD [4] and the timely transfer of PD patients to HD is important for long-term survival [5]. However, as bridge therapy between full-time PD and full-time HD, the role of combined PD and HD therapy (combined therapy) is always overlooked.

Combined therapy was first proposed in 1996 by Kimura and Watanabe in Japan [6]. Due to their advantages, this therapy, generally comprising 5-6 days of PD and one HD session per week, overcomes the shortcomings of PD and HD and is more acceptable than direct transfer to HD. Several studies showed that combined therapy could increase dialysis adequacy, decrease fluid overload, improve peritoneal membrane function, and ultimately boost quality of life [6-9]. However, these single-center studies were limited by small patient numbers and focused on before versus after comparisons. One single-center study indicated an equivalent mortality risk for combined therapy, HD alone, and PD alone [10].

The Taiwanese National Health Insurance (TNHI) system provides services for $\mathrm{HD}$ and $\mathrm{PD}$, including the use of icodextrin, Nutrineal, and automated PD. The TNHI system has also expanded coverage to combined therapy, but only if the symptoms of uremia and fluid overload cannot be ameliorated by maximizing the PD prescription. PD patients can decide whether to directly transfer to HD or to receive combined therapy.

To the best of our knowledge, our investigation is the first nationwide study of combined therapy. The present study aimed to explore the current practice of combined therapy and to clarify the long-term prognosis for mortality and admission risks if PD patients select it as bridge therapy.

\section{Methods}

\section{Data sources}

We used a longitudinal health insurance database of catastrophic illness patients (LHID-CIP) for this retrospective cohort study. The LHID-CIP was obtained from the TNHI program released by the Taiwanese National Health Insurance Administration, Ministry of Health and Welfare. Thirty disease categories were included in the registry of catastrophic illness patients in Taiwan, including malignancy, coagulation defects, end-stage renal disease (ESRD), and type I diabetes. Based on TNHI guidelines, a patient was defined as a catastrophic illness patient (CIP) by a specialist and by clinical reports such as pathological reports, blood tests, and kidney function tests. The LHID-CIP contained medical claims and patient information from 1997 to 2011. LHID-CIP diseases were defined according to the International Classification of Diseases, Ninth Revision, Clinical Modification (ICD-9$\mathrm{CM}$ ), which was established by the World Health Organization. To protect personal information, this study was approved by the Research Ethics Committee at China Medical University and Hospital (CMUH104REC2-115-CR-4).

\section{Study participants}

All 12,407 ESRD patients (ICD-9-CM code 585) who received PD from 2000 to 2010 were collected. Patients aged $<18$ years were excluded, as well as those with a history of kidney transplantation, stayed on PD, and death or kidney transplantation on PD. The patients were divided into two groups: a combined therapy group (combined group) and a group comprising those directly transferred to HD (transfer group). Patients who were undergoing regular PD with at least two HD sessions per month at the outpatient department were included in the combined group. Patients who stopped PD and permanently transferred to $\mathrm{HD}$ treatment at the outpatient department were included in the transfer group. For added comparability, the transfer group was frequency matched with the combined group according to age, sex, and duration on PD (time from PD to HD initiation). The date of HD initiation was defined as the index date. The details are shown in Supplementary Figure 1.

\section{Outcomes and risk factors}

The outcomes included mortality and incidence of admission. Admission included both hospitalization and emergency department visit. All participants were followed up from the index date until the date of mortality or admission. Those without outcome occurrence were followed up until the date of withdrawal from the TNHI program or the end of 2011. Risk factors considered in this study included age, sex, year of PD, duration on PD, Charlson comorbidity index, baseline comorbidity (including diabetes, hypertension, ischemic heart disease, chronic heart failure, cerebrovascular disease, peripheral arterial occlusive disease, and malignancy), medical treatment use (including automated PD and icodextrin use within 3 months before the index date), recent peritonitis (peritonitis diagnosis within 3 months before the index date), and type of vascular access for HD (including arteriovenous fistula/arteriovenous graft and tunneled catheter) before initiation of hemodialysis. Temporary double lumen catheter was used for hemodialysis by patients who did not have prepared vascular access. 


\section{Reasons for admission}

Reasons for admission were divided into peritonitis (ICD-9-CM codes 996.68, 567.9, and 567.2), infection due to vascular devices, implants, and grafts (ICD-9-CM code 996.62), infection other than peritonitis and vascular devices (ICD-9-CM codes 999.3, 599.0, and 486), other complications of PD (ICD-9-CM codes V562, 996.56, and 550.90), other complications of vascular devices (ICD-9-CM codes 996.1 and 996.73), and coronary artery disease (ICD-9-CM codes 414.01, 428.0, and 518.4).

\section{Statistical analysis}

All statistical analyses were performed using SAS software version 9.4 (SAS Institute, Cary, NC), and the statistical significance level was set at $p<0.05$ with a two-tailed test. Categorical variables are presented as number and percentage. Differences between the two groups were determined using a chi-square test or Fisher's exact test when the cell number was less than five. Continuous variables are shown as the mean (standard deviation [SD]) and median (quartile 1 [Q1] and quartile 3 [Q3]), and differences between the two groups were determined by Student's $t$-test and Wilcoxon rank-sum test. The mortality and incidence of admission were calculated according to the sum of the outcome number (death or admission) divided by the sum of person-years in both groups. A standard Cox proportional hazards model was used to assess the risk of death. Because the mortality differed between the two groups, we used a Cox proportional hazards model with competing risk of death to estimate the risk of admission. A multivariable Cox model was adjusted for age, sex, and variables with significant differences, as shown in Table 1. The stratified analysis was adjusted by age, duration of PD, Charlson comorbidity index, and recent peritonitis. Cumulative incidence of death and admission was plotted by KaplanMeier analysis, and a log-rank test was used to evaluate differences between the two groups. The distribution of the duration of PD and duration on hybrid therapy in the combined group was also presented. The propensity score matching method was used for sensitivity analysis in this study. The propensity score of the combined group and transfer group was calculated using stepwise logistic regression models which involved age, Charlson comorbidity index score, DM, use of icodextrin, type of vascular access for $\mathrm{HD}$, and recent peritonitis.

\section{Results}

From 2000 to 2010, 691 PD patients choose combined therapy as bridge therapy. The hemodialysis frequency per month was two (350 patients), three (28 patients), or four (310 patients). The numbers of incidents in the combined therapy patients were 4 in 2000, 81 in 2006, and 138 in 2010 (data not shown). The highest proportion of combined therapy was shown in the first year of
PD (29.2\%), and the proportion decreased in the year after PD (Supplementary Figure 2A). Calculation of the duration between the onset of combined therapy to the end, including pure $\mathrm{HD}$, death, kidney transplantation, or the end of follow-up, revealed that about $42 \%$ of patients were maintained on combined therapy for less than 1 year and that patients maintained combined therapy for an average of 2.05 years $(S D=2.00)$ (Supplementary Figure 2B).

After frequency matching, 688 patients from the combined group and 688 patients from the transfer group were selected in this study. The mean age was 50.2 years $(\mathrm{SD}=14.3)$ and there were slightly more males than females (50.4\% vs. $49.6 \%)$ (Table 1$)$. Compared with the transfer group, the combined group had a higher Charlson comorbidity index $(p<0.0001)$ but less commonly had diabetes $(36.9 \%$ vs. $45.6 \%, p=0.001)$, recent peritonitis $(31.0 \%$ vs. $50.0 \%, p<0.0001)$, and vascular access for HD (65.8\% vs. $84.9 \%, p<0.0001)$. Patients from combined group were further divided into two groups by hemodialysis frequency $(\mathrm{HD} \leq 3$ and $\mathrm{HD}=4$ ) (Table $1)$. Compared with $\mathrm{HD} \leq 3$ group, $\mathrm{HD}=4$ group had more females (54.8\% vs. $45.2 \%)$, a higher usage of APD (27.4\% vs. $19.1 \%)$, usage of icodextrin $(47.1 \%$ vs. $38.1 \%)$, recent peritonitis ( $38.1 \%$ vs. $25.1 \%)$, use of tunneled catheter as vascular access for HD (32.3\% vs. 8.73\%).

During a 12-year follow-up, 145 and 155 patients died in the combined and transfer groups, giving mortality rates of 60.09 and 68.68 per 1000 person-years, respectively. Although the cumulative incidence of death was higher in the transfer group than in the combined group, it was not significantly different (Fig. 1a). Compared with the transfer group, the combined group had a similar risk of death (hazard ratio $[\mathrm{HR}]=1.02,95 \%$ confidence interval $[\mathrm{CI}]=0.80-1.30$ ) in a Cox proportional hazards model after adjustment for age, sex, diabetes, chronic heart failure, Charlson comorbidity index, recent peritonitis, and type of vascular access for HD (Table 2). When the combined group was stratified by frequency of HD, the risk of death was unchanged. All 646 and 628 patients exhibited an admission event, and the corresponding incidences were 1969.17 and 1656.15 per 1000 person-years in the combined and transfer groups, respectively. The cumulative incidence of admission was similar in the two groups according to Kaplan-Meier analysis (Fig. 1b). In a multivariable Cox proportional hazards model with competing risk of death, a similar risk of admission was found in the two groups $(\mathrm{HR}=$ 1.06, 95\% CI =0.95-1.19) (Table 2). Compared with the transfer group, combined group patients with four HD sessions per month had higher risk of admission $(\mathrm{HR}=$ 1.18, 95\% CI =1.03-1.35). Furthermore, we performed sensitivity analyses with propensity score matching method to compare the prognosis between combined 
Table 1 Demographic profiles of peritoneal patients in the combined and transfer groups

\begin{tabular}{|c|c|c|c|c|c|c|c|c|c|c|}
\hline \multirow[b]{3}{*}{ Variable } & \multicolumn{6}{|c|}{ Combined group } & \multirow{2}{*}{\multicolumn{2}{|c|}{$\begin{array}{l}\text { Transfer } \\
\text { group } \\
(N=688)\end{array}$}} & \multirow{3}{*}{$\begin{array}{l}\text { Combined group vs. } \\
\text { Transfer group } \\
\text { p-value }\end{array}$} & \multirow{3}{*}{$\begin{array}{l}\text { Three } \\
\text { groups } \\
p \text {-value } \\
\end{array}$} \\
\hline & \multicolumn{2}{|c|}{$\begin{array}{l}H D^{a} \leq 3 \\
(N=378)\end{array}$} & \multicolumn{2}{|c|}{$\begin{array}{l}H^{a}=4 \\
(N=310)\end{array}$} & \multicolumn{2}{|c|}{$\begin{array}{l}\text { Total } \\
(N=688)\end{array}$} & & & & \\
\hline & $n$ & $\%$ & $n$ & $\%$ & $n$ & $\%$ & $n$ & $\%$ & & \\
\hline Age, years & & & & & & & & & 1.00 & 0.67 \\
\hline$<65$ & 316 & 83.6 & 251 & 81.0 & 567 & 82.4 & 567 & 82.4 & & \\
\hline$\geq 65$ & 62 & 16.4 & 59 & 19.3 & 121 & 17.6 & 121 & 17.6 & & \\
\hline Mean (SD) & 49.1 & $(14.3)$ & 51.6 & $(14.2)$ & 50.2 & (14.3) & 50.2 & $(14.3)$ & 0.91 & 0.08 \\
\hline Sex & & & & & & & & & 1.00 & 0.04 \\
\hline Female & 171 & 45.2 & 170 & 54.8 & 341 & 49.6 & 341 & 49.6 & & \\
\hline Male & 207 & 54.8 & 140 & 45.2 & 347 & 50.4 & 347 & 50.4 & & \\
\hline Year at cohort entry & & & & & & & & & 0.38 & $<0.0001$ \\
\hline Before 2004 & 114 & 30.2 & 15 & 4.84 & 129 & 18.8 & 142 & 20.6 & & \\
\hline After 2004 & 264 & 69.8 & 295 & 95.2 & 559 & 81.3 & 546 & 79.4 & & \\
\hline Duration of peritoneal dialysis, years & & & & & & & & & 1.00 & 0.11 \\
\hline$<1$ year & 120 & 31.8 & 81 & 26.1 & 201 & 29.2 & 201 & 29.2 & & \\
\hline $1-2$ years & 82 & 21.7 & 68 & 21.9 & 150 & 21.8 & 150 & 21.8 & & \\
\hline $2-5$ years & 144 & 38.1 & 111 & 35.8 & 255 & 37.1 & 255 & 37.1 & & \\
\hline$>5$ years & 32 & 8.47 & 50 & 16.1 & 82 & 11.9 & 82 & 11.9 & & \\
\hline Mean (SD) & 2.32 & $(1.96)$ & 2.72 & $(2.17)$ & 2.50 & (2.06) & 2.53 & $(2.17)$ & 0.83 & 0.04 \\
\hline \multicolumn{11}{|l|}{ Charlson comorbidity index } \\
\hline Median (Q1, Q3) & 3 & $(2,4)$ & 3 & $(2,4)$ & 3 & $(2,4)$ & 3 & $(2,4)$ & $<0.0001$ & $<0.0001$ \\
\hline Mean (SD) & 3.04 & $(1.76)$ & 2.96 & $(1.78)$ & 3.63 & $(1.77)$ & 3.01 & $(1.77)$ & 0.0002 & 0.0009 \\
\hline \multicolumn{11}{|l|}{ Comorbidity } \\
\hline Diabetes & 137 & 36.2 & 117 & 37.7 & 254 & 36.9 & 314 & 45.6 & 0.001 & 0.004 \\
\hline Hypertension & 360 & 95.2 & 297 & 95.8 & 657 & 95.5 & 656 & 95.4 & 0.90 & 0.93 \\
\hline Ischemic heart disease & 124 & 32.8 & 114 & 36.8 & 238 & 34.6 & 257 & 37.4 & 0.29 & 0.32 \\
\hline Chronic heart failure & 88 & 23.3 & 74 & 23.9 & 162 & 23.6 & 194 & 28.2 & 0.049 & 0.14 \\
\hline Cerebrovascular disease & 67 & 17.7 & 58 & 18.7 & 125 & 18.2 & 142 & 20.6 & 0.25 & 0.48 \\
\hline PAOD & 27 & 7.14 & 27 & 8.71 & 54 & 7.85 & 53 & 7.70 & 0.92 & 0.74 \\
\hline Malignancy & 24 & 6.35 & 23 & 7.42 & 47 & 6.83 & 48 & 6.98 & 0.92 & 0.85 \\
\hline Use of APD & 72 & 19.1 & 85 & 27.4 & 157 & 22.8 & 173 & 25.2 & 0.31 & 0.02 \\
\hline Use of icodextrin & 144 & 38.1 & 146 & 47.1 & 290 & 42.2 & 282 & 41.0 & 0.66 & 0.053 \\
\hline Recent peritonitis & 95 & 25.1 & 118 & 38.1 & 213 & 31.0 & 344 & 50.0 & $<0.0001$ & $<0.0001$ \\
\hline Type of vascular access for HD & & & & & & & & & $<0.0001$ & $<0.0001$ \\
\hline AVF/AVG & 205 & 54.2 & 115 & 37.1 & 320 & 46.5 & 425 & 61.8 & & \\
\hline Tunneled catheter & 33 & 8.73 & 100 & 32.3 & 133 & 19.3 & 159 & 23.1 & & \\
\hline
\end{tabular}

$A V F$ arteriovenous fistula, $A V G$ arteriovenous graft, $A P D$ automated $P D, P A O D$ peripheral artery occlusive disease

${ }^{a} H D$ hemodialysis sessions per month

group and transfer group (Supplementary Table 1) (Supplementary Table 2). In this analysis, outcome was similar to the primary analysis but there was no difference in risk of admission or mortality when the combined group was stratified by frequency of HD.

Figure 2 presents the associations between outcomes and groups stratified by age, duration of PD, Charlson comorbidity index, and recent peritonitis. For admission events, the combined group exhibited a similar trend in the different subgroups compared with the transfer group, except in patients with recent peritonitis. In those with recent peritonitis, the combined group had significantly higher risk of admission than the transfer group ( $\mathrm{HR}=1.65,95 \% \mathrm{CI}=1.37-1.99)$. However, in those without recent peritonitis, the combined group had significantly lower risk than the transfer group $(\mathrm{HR}=$ 

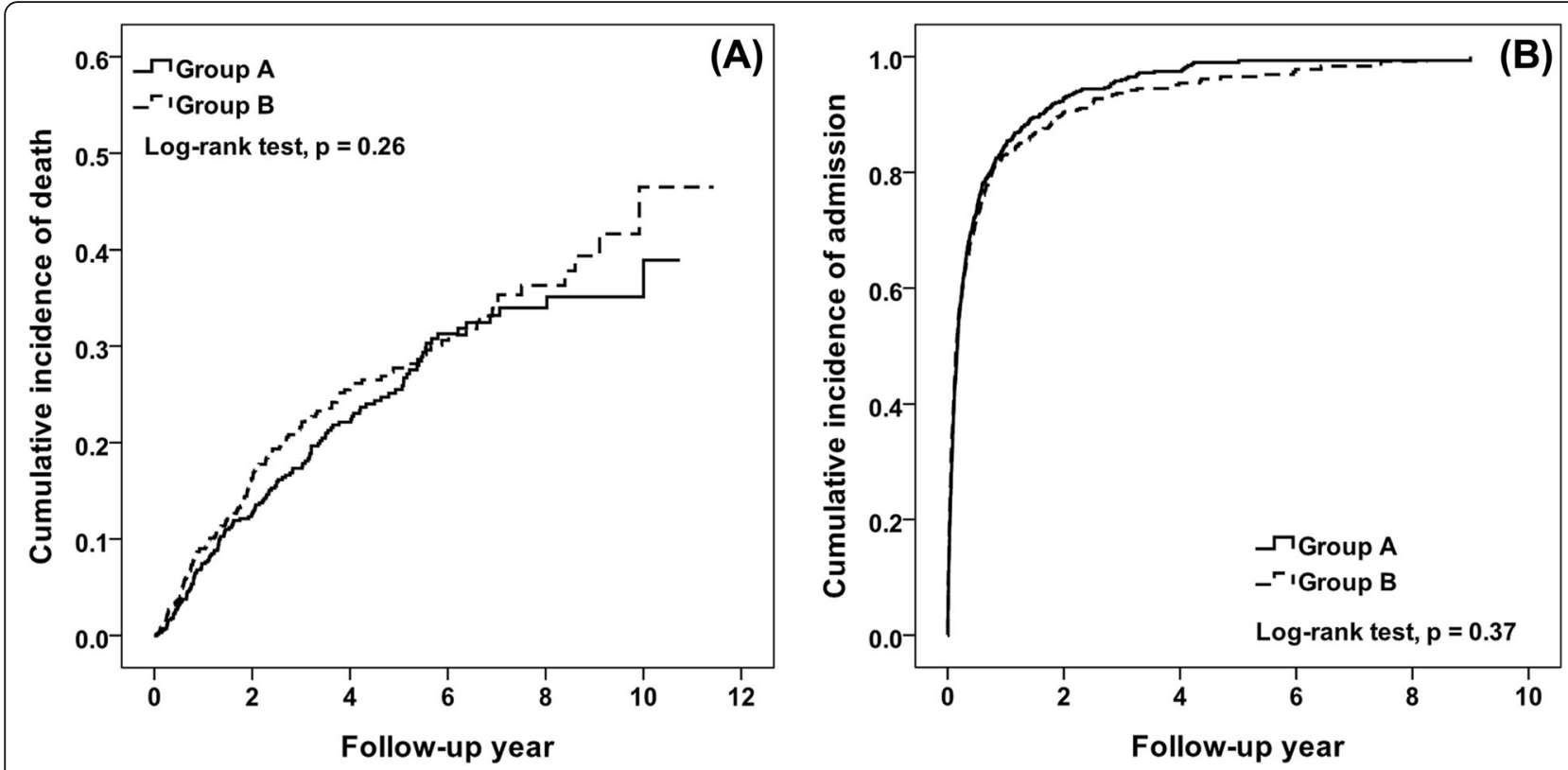

Fig. 1 a Cumulative mortality risk and (b) admission risk of patients on combined therapy (combined group) and those who directly transferred to HD (transfer group)

0.82, 95\% CI $=0.71-0.95$ ) (interaction $p<0.0001$ ). The different subgroups of the two groups had similar mortality rates (interaction $p>0.05$ ).

Table 3 presents the incidence rate ratios of different reasons for admission between two groups. Compared with the transfer group, peritonitis was the main reason for admission and was significantly more common in the combined group after adjustment $(\mathrm{HR}=2.06,95 \% \mathrm{CI}=$ 1.23-3.46) but admission for infection due to vascular devices, implants, and grafts was significantly lower in the combined group $(\mathrm{HR}=0.46,95 \% \mathrm{CI}=0.23-0.92)$.

\section{Discussion}

This 12-year nationwide population-based follow-up study obtained several significant findings from patients receiving combined therapy. First, the overall mortality and admission risks of these patients were comparable to those who directly transferred to HD. Furthermore, in

Table 2 Incidence rate ratios of admission and mortality risk according to HD frequency in the combined and transfer groups

\begin{tabular}{|c|c|c|c|c|c|}
\hline Outcome & Event & Person-years & Rate & SHR $(95 \% \mathrm{Cl})$ & $p$-value \\
\hline \multicolumn{6}{|l|}{ Admission } \\
\hline \multicolumn{6}{|l|}{ Combined group } \\
\hline Overall $(N=688)$ & 646 & 328 & 1969.17 & $1.06(0.95-1.19)$ & 0.32 \\
\hline HD sessions $=2(N=350)$ & 328 & 209 & 1570.58 & $0.96(0.84-1.10)$ & 0.56 \\
\hline HD sessions = $3(N=28)$ & 26 & 13 & 2059.98 & $1.11(0.74-1.66)$ & 0.61 \\
\hline HD sessions $=4(N=310)$ & 292 & 107 & 2739.33 & $1.18(1.03-1.35)$ & 0.02 \\
\hline Transfer group & 628 & 379 & 1656.15 & Ref. & \\
\hline Outcome & Event & Person-years & Rate & HR $(95 \% \mathrm{Cl})$ & $p$-value \\
\hline \multicolumn{6}{|l|}{ Mortality } \\
\hline \multicolumn{6}{|l|}{ Combined group } \\
\hline Overall $(N=688)$ & 145 & 2413 & 60.09 & $1.02(0.80-1.30)$ & 0.88 \\
\hline HD sessions $=2(N=350)$ & 79 & 1430 & 55.23 & $1.02(0.77-1.37)$ & 0.88 \\
\hline HD sessions = $3(N=28)$ & 6 & 116 & 51.66 & $1.31(0.57-2.99)$ & 0.52 \\
\hline HD sessions $=4(N=310)$ & 60 & 867 & 69.24 & $1.00(0.73-1.36)$ & 0.98 \\
\hline Transfer group & 155 & 2257 & 68.68 & Ref. & \\
\hline
\end{tabular}

Rate, per 1000 person-years; SHR subdistribution HR

Adjusted for age, sex, diabetes, chronic heart failure, Charlson comorbidity index, recent peritonitis, and type of vascular access for HD 


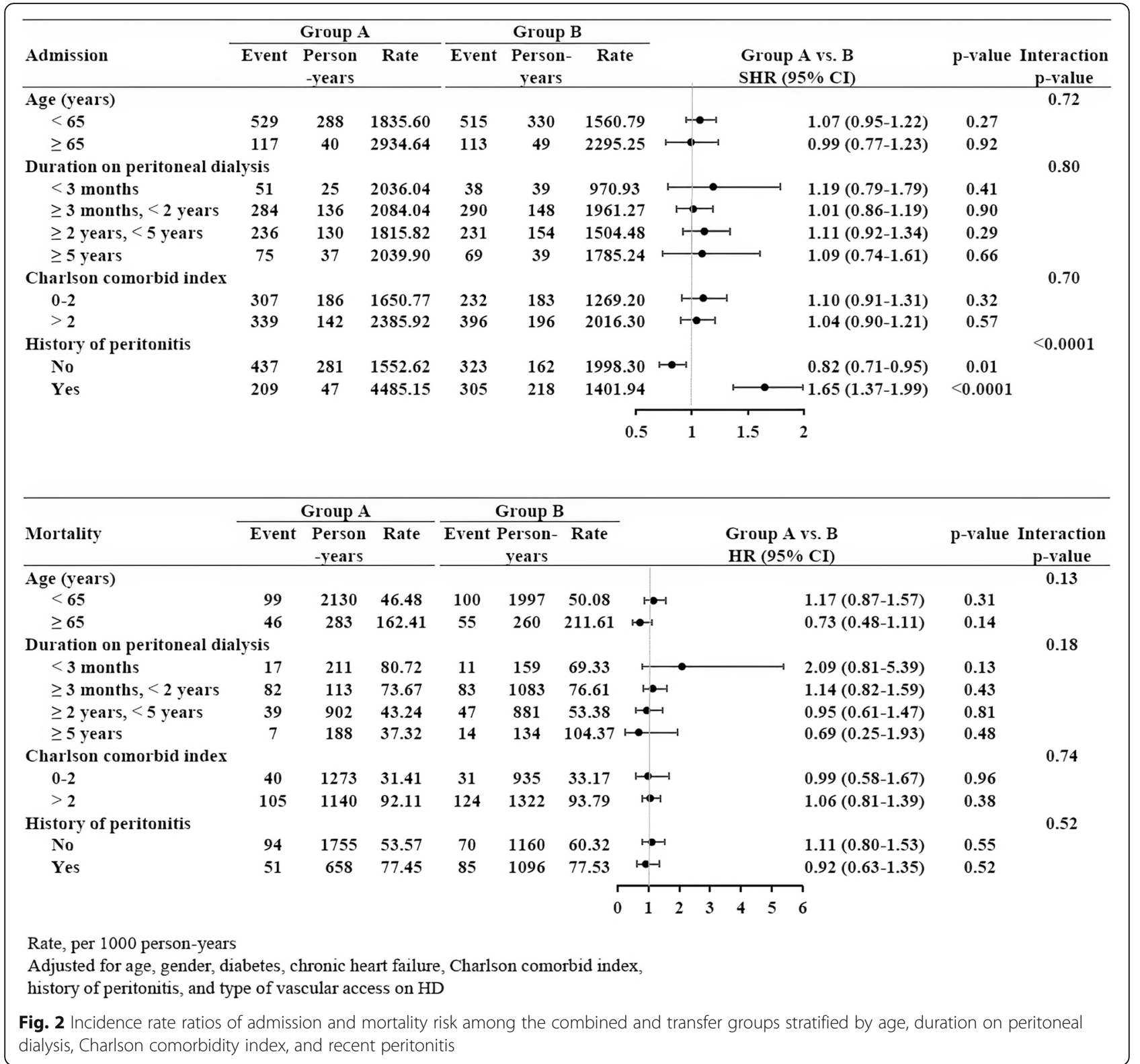

Table 3 Incidence rate ratios of different reasons for admission between the combined and transfer groups

\begin{tabular}{|c|c|c|c|c|c|c|c|c|}
\hline \multirow[t]{2}{*}{ Outcome } & \multicolumn{3}{|c|}{ Combined group } & \multicolumn{3}{|c|}{ Transfer group } & \multirow[t]{2}{*}{ SHR $(95 \% \mathrm{Cl})$} & \multirow[t]{2}{*}{$p$-value } \\
\hline & Event & Person-years & Rate & Event & Person-years & Rate & & \\
\hline Peritonitis & 49 & 328 & 149.36 & 25 & 379 & 65.93 & $2.06(1.23-3.46)$ & 0.006 \\
\hline $\begin{array}{l}\text { Infection due to vascular devices, } \\
\text { implants, and grafts }\end{array}$ & 13 & 328 & 39.63 & 27 & 379 & 71.20 & $0.46(0.23-0.92)$ & 0.03 \\
\hline $\begin{array}{l}\text { Infection other than peritonitis } \\
\text { and vascular devices }\end{array}$ & 31 & 328 & 94.50 & 26 & 379 & 68.57 & $1.22(0.66-2.26)$ & 0.53 \\
\hline Other complications of PD & 56 & 328 & 170.70 & 49 & 379 & 129.22 & $0.88(0.57-1.36)$ & 0.56 \\
\hline $\begin{array}{l}\text { Other complications of vascular } \\
\text { devices }\end{array}$ & 23 & 328 & 70.11 & 39 & 379 & 102.85 & $0.69(0.40-1.21)$ & 0.20 \\
\hline CAD & 23 & 328 & 70.11 & 18 & 379 & 47.47 & $1.52(0.79-2.95)$ & 0.21 \\
\hline
\end{tabular}

CAD coronary artery disease

Rate, per 1000 person-years. SHR subdistribution HR

Adjusted for age, sex, diabetes, chronic heart failure, Charlson comorbidity index, recent peritonitis, and type of vascular access for HD 
subgroup analysis, patients without recent peritonitis benefited from combined therapy due to lower admission risk. Finally, subsequent peritonitis was a major risk factor for admission. Combined therapy with two HD sessions per month was shown to be a good therapeutic option.

In the United States, conversion from PD to HD is frequent and occurs at a mean annual rate of 9.5\% [4]. In a previous study, peritonitis was the most common cause of technique failure (average, 43\%), followed by inadequate dialysis/ultrafiltration (average, 23\%) [11]. Although we were unable to identify the actual cause of technique failure in our study, it can be deduced from our data on recent peritonitis and the PD regimen. Peritonitis (31 and 50\% in the combined and transfer groups, respectively) and ultrafiltration failure were the causes of technique failure because icodextrin and automated PD were used by approximately 40 and $20 \%$ of patients in each group [12].

PD patients who experience technique failure could benefit both physically and psychologically from combined therapy. Physically, combined therapy improves dialysis adequacy and fluid overload. It also decreases body weight, blood pressure, serum creatinine, and even left ventricular mass index [13], as well as increases serum hemoglobin. Combined therapy may improve peritoneal membrane function by allowing a "PD holiday" entailing peritoneal rest [14]. Psychologically, combined therapy has a positive effect on health-related quality of life, including better physical function, fewer symptoms of kidney disease, and less of an impact of kidney disease on daily life [8]. It should also be emphasized that combined therapy may be more acceptable to patients because it permits a minimal lifestyle change and home-based continuous therapy.

When physicians are informing patients of technique failure and facing the transition, shared decision-making to determine individual preferences is important. It is necessary to explain the differences in the long-term outcomes of combined therapy and direct transfer to HD. Our study provides strong evidence of similar mortality and admission risks in the two groups after adjustment for potential confounders. Furthermore, we evaluated "life quality", reflected by admission risk, because hospitalization is often followed by a decline in functional status that affects quality of life [15], and it appeared to be similar in the combined therapy and HD transfer groups.

For patients who transferred from PD to HD, the mortality risk peaked in the first year, which was attributed to infection (recurrent peritonitis/arteriovenous access infection) and cardiovascular events [16]. In our study, combined therapy was associated with a significantly higher peritonitis rate but lower vascular access infection than direct transfer to HD. It is worth emphasizing that peritonitis was the main negative factor for combined therapy. Not only was peritonitis associated with high admission risk, but also subsequent peritonitis was the main reason for admission in combined therapy patients. Recent peritonitis might increase the risk of repeat peritonitis [17] and aggravate ultrafiltration failure and membrane-related problems, which could partly explain the higher admission risk. We suggest that patients with recent peritonitis or high risk for peritonitis might be discouraged from combined therapy.

Because of the similar prognosis of combined therapy and transfer to HD, cost-effectiveness analysis is essential. For combined therapy, two HD sessions per month were covered by the TNHI system, but the other two sessions need to be paid by the patients themselves. Previous work reported PD and HD costs (US\$/per year) in Taiwan of 17,723 and 21,367, respectively, including outpatient and inpatient expenses [18]. Each HD session costs $\$ 133$ in Taiwan. Thus, combined therapy with two HD sessions per month costs $\$ 21,192$ per year (26 HD sessions per year), which is still lower than the cost of pure HD; in contrast, combined therapy with four HD sessions per month is more expensive than pure HD. From insurance aspect, combined therapy with two HD sessions per month was an acceptable choice.

There is no doubt that four HD sessions per month were better for dialysis adequacy than two HD sessions per month, and the latter sometimes was regarded as rescue HD not combined therapy. Combined therapy with four HD sessions was unexpectedly associated with higher admission risk in our study, which might be not only related to complications with frequent hemodialysis, but also reflect the underlined difference between these two groups of patients. Compared to two HD sessions, combined group with four HD sessions had more usage of APD, icodextrin, recent peritonitis and more tunneled catheter as vascular access to HD. Combined therapy with two HD sessions per month was a feasible alternative from clinical and insurance perspective in Taiwan.

Given the advantages of combined therapy, the total number of patients in Japan on combined therapy increased from 600 in 2002 (5.5\% of all PD patients) to 1900 patients in 2013 (20\% of all PD patients) [10]. The use of combined therapy has increased in recent years in Taiwan but is still considerably lower than in Japan. Our dialysis staff should be familiar with the advantages and disadvantages of combined therapy and consider it an essential part of integrated dialysis care.

The strengths of this study include its nationwide scope and the fact that Taiwan is one of the few countries to adopt combined therapy. Furthermore, given the high coverage rate and continuity of the TNHI database, we were able to compare long-term outcomes between 
combined therapy and direct transfer to HD. Our findings are essential to determine the final piece in the puzzle of integrated dialysis care.

Some limitations of this study should also be considered. First, detailed laboratory results were not included in our database. We could not identify the albumin level, residual renal function, nutritional status, ultrafiltration rate, peritoneal function test, plasma $\beta 2$-microglobulin level, hyperphosphatemia, and dialysis clearance (weekly $\mathrm{Kt} / \mathrm{V}$ and weekly creatinine clearance), all of which might be associated with the outcome. Second, despite age, sex, and PD duration being matched between patients with combined therapy and those who transferred to HD, there were still residual biases between the groups, including indications and numerical data of changing the dialysis modality to combined or transfer therapy, fluid status, poor self-management of fluid balance, possibility of continuing PD therapy and the patients' requests. Third, the combined therapy protocol, including the numbers of HD sessions per month and the length of PD holidays, might have varied among hospitals.

\section{Conclusions}

Our study revealed that combined therapy is not a redundant but a rational and cost-effective therapy, particularly for patients without recent peritonitis. This study aimed to support evidence-based shared decision-making for PD patients facing the transition. Dialysis unit staff, including physicians and nurses, should be able to introduce combined therapy to patients as an ideal alternative choice.

\section{Supplementary information}

Supplementary information accompanies this paper at https://doi.org/10 1186/s12882-020-01989-1.

Additional file 1: Table S1. Demographic profiles of peritoneal patients in the combined and transfer groups by propensity score matching method. Table S2. Incidence rate ratios of admission and mortality risk according to HD frequency in the combined and transfer groups by propensity score matching method.

Additional file 2: Figure S1. Flow diagram illustrating patient selection. Additional file 3: Figure S2. (A) Distribution of the duration of PD at the start of combined therapy (years). (B) Distribution of the duration between the onset of combined therapy to the end, including transfer to $H D$, death, kidney transplantation, or the end of follow-up (years).

\section{Abbreviations}

PD: Peritoneal dialysis; HD: Hemodialysis; ICD-9-CM: International Classification of Diseases Ninth Revision Clinical Modification; HR: Hazard ratio

\section{Acknowledgements}

Not Applicable.

\section{Authors' contributions}

MCC, TMY, MJW, YWC, CHC1, CHC2, JJS, PHH, JWC, and CJC designed the research. MCC, CHM, and CJC analyzed and interpreted the data. MCC, CHM, and $\mathrm{CJC}$ wrote the first draft of the paper. All authors participated in revising the paper. The author(s) read and approved the final manuscript.

\section{Funding}

This study is supported in part by Taiwan Ministry of Health and Welfare Clinical Trial Center (MOHW109-TDU-B-212-114004), Taichung Veterans General Hospital (TCVGH-1073601B and TCVGH-1083602B), Cheng Shiu University, Kaohsiung, Taiwan, MOST Clinical Trial Consortium for Stroke (MOST 108-2321-B-039-003), Tseng-Lien Lin Foundation, Taichung, Taiwan. The funders performed no role in the study design, data collection and analysis, decision to publish, or manuscript preparation.

\section{Availability of data and materials}

The data that support the findings of this study are available from the Taiwan National Health Insurance Research Database (NHIRD) but restrictions apply to the availability of these data, which were used under license for the current study, and so are not publicly available. Any detail for data requests can be through the NHIRD (http://nhird.nhri.org.tw).

\section{Ethics approval and consent to participate}

This study was approved by the Institutional Review Board of the China Medical University Hospital (CMUH104-REC2-115(CR-4)) in Taiwan.

\section{Consent for publication}

Not applicable.

\section{Competing interests}

The authors declare that they have no competing interests.

\section{Author details}

${ }^{1}$ Division of Nephrology, Department of Medicine, Taichung Veterans General Hospital, Taichung, Taiwan. ${ }^{2}$ Program in Translational Medicine, National Chung Hsing University, Taichung, Taiwan. ${ }^{3}$ Rong Hsing Research Center for Translational Medicine, National Chung Hsing University, Taichung, Taiwan. ${ }^{4}$ Management Office for Health Data, China Medical University and Hospital, Taichung, Taiwan. ${ }^{5}$ Department of Urology, China Medical University and Hospital, Taichung, Taiwan. ${ }^{6}$ Department of Medicine, College of Medicine, China Medical University and Hospital, Taichung, Taiwan. ${ }^{7}$ Institute of Biomedical Sciences, National Chung Hsing University, Taichung, Taiwan. ${ }^{8}$ Department of Education and Research, Taichung Veterans General Hospital, Taichung, Taiwan. ${ }^{9}$ Department of Internal Medicine, Ditmanson Medical Foundation Chiayi Christian Hospital, Chiayi, Taiwan. ${ }^{10}$ Department of Applied Life Science and Health, Chia-Nan University of Pharmacy and Science, Tainan, Taiwan. ${ }^{11}$ Department of Food and Beverage Management, Cheng Shiu University, Kaohsiung, Taiwan. ${ }^{12}$ Center for Environmental Toxin and Emerging-Contaminant Research, Cheng Shiu University, Kaohsiung, Taiwan. ${ }^{13}$ Super Micro Mass Research and Technology Center, Cheng Shiu University, Kaohsiung, Taiwan. ${ }^{14}$ Department of Public Health, College of Public Health, China Medical University, No. 91 Hsueh-Shih Road, Taichung 40402, Taiwan. ${ }^{15}$ Department of Medical Research, China Medical University Hospital, Taichung, Taiwan.

Received: 14 January 2020 Accepted: 29 July 2020

Published online: 15 August 2020

\section{References}

1. Kumar VA, Sidell MA, Jones JP, Vonesh EF. Survival of propensity matched incident peritoneal and hemodialysis patients in a United States health care system. Kidney Int. 2014;86:1016-22.

2. Sinnakirouchenan R, Holley JL. Peritoneal dialysis versus hemodialysis: risks, benefits, and access issues. Adv Chronic Kidney Dis. 2011;18:428-32.

3. Blake PG. Integrated end-stage renal disease care: the role of peritoneal dialysis. Nephrol Dial Transplant. 2001;16(Suppl 5):61-6.

4. Chaudhary K, Sangha H, Khanna R. Peritoneal dialysis first: rationale. Clin J Am Soc Nephrol. 2011;6:447-56.

5. Panagoutsos S, Kantartzi K, Passadakis P, Yannatos E, Mourvati E, Theodoridis M, Kriki $P$, Thodis E, Vargemezis $V$. Timely transfer of peritoneal dialysis patients to hemodialysis improves survival rates. Clin Nephrol. 2006;65:43-7.

6. Fukui $\mathrm{H}$, Hara S, Hashimoto Y, Horiuchi T, Ikezoe M, Itami N, Kawabe M, Kawanishi $H$, Kimura $H$, Nakamoto $Y$, et al. Review of combination of peritoneal dialysis and hemodialysis as a modality of treatment for endstage renal disease. Ther Apher Dial. 2004;8:56-61.

7. Maruyama Y. Does combined therapy with peritoneal Dialysis and hemodialysis improve prognosis? Contrib Nephrol. 2018;196:64-70. 
8. Watanabe $\mathrm{Y}$, Okada $\mathrm{H}$. Effect of combined peritoneal Dialysis and hemodialysis on health-related quality of life. Contrib Nephrol. 2018; 196:135-40

9. Maruyama Y, Yokoyama K, Nakayama M, Higuchi C, Sanaka T, Tanaka Y, Sakai K, Mizuiri S, Otsuka Y, Kuriyama S, et al. Combined therapy with peritoneal dialysis and hemodialysis: a multicenter retrospective observational cohort study in Japan. Blood Purif. 2014;38:149-53.

10. Matsuo N, Yokoyama K, Tanno Y, Yamamoto I, Yokoo T. Combined therapy using peritoneal dialysis and hemodialysis may increase the indications for peritoneal dialysis in the United States. Kidney Int. 2015;87:1259-60.

11. Gallieni M, Giordano A, Ricchiuto A, Gobatti D, Cariati M. Dialysis access: issues related to conversion from peritoneal dialysis to hemodialysis and vice versa. J Vasc Access. 2017;18:41-6.

12. Krediet $R$, Mujais $S$. Use of icodextrin in high transport ultrafiltration failure Kidney Int Suppl. 2002;62:S53-61.

13. Tanaka M, Mise N, Nakajima H, Uchida L, Ishimoto Y, Kotera N, Tanaka $\mathrm{S}$, Kurita N, Sugimoto T. Effects of combination therapy with peritoneal dialysis and hemodialysis on left ventricular hypertrophy. Perit Dial Int. 2011;31:598-600.

14. Kawanishi H, Moriishi M. Clinical effects of combined therapy with peritoneal dialysis and hemodialysis. Perit Dial Int. 2007;27(Suppl 2):S126-9.

15. Admi H, Shadmi E, Baruch $H$, Zisberg A. From research to reality: minimizing the effects of hospitalization on older adults. Rambam Maimonides Med J. 2015;6:e0017.

16. Szeto CC, Kwan BC, Chow KM, Pang WF, Kwong WW, Leung CB, Li PK. Outcome of hemodialysis patients who had failed peritoneal dialysis. Nephron Clin Pract. 2010;116:c300-6.

17. Thirugnanasambathan T, Hawley CM, Badve SV, McDonald SP, Brown FG, Boudville N, Wiggins KJ, Bannister KM, Clayton P, Johnson DW. Repeated peritoneal dialysis-associated peritonitis: a multicenter registry study. Am J Kidney Dis. 2012;59:84-91.

18. Chang YT, Hwang JS, Hung SY, Tsai MS, Wu JL, Sung JM, Wang JD. Costeffectiveness of hemodialysis and peritoneal dialysis: a national cohort study with 14 years follow-up and matched for comorbidities and propensity score. Sci Rep. 2016;6:30266.

\section{Publisher's Note}

Springer Nature remains neutral with regard to jurisdictional claims in published maps and institutional affiliations.

Ready to submit your research? Choose BMC and benefit from:

- fast, convenient online submission

- thorough peer review by experienced researchers in your field

- rapid publication on acceptance

- support for research data, including large and complex data types

- gold Open Access which fosters wider collaboration and increased citations

- maximum visibility for your research: over $100 \mathrm{M}$ website views per year

At $\mathrm{BMC}$, research is always in progress.

Learn more biomedcentral.com/submissions 